\title{
On the Regime of Ground Water during Filtration from Channels in the Soil Layer with the Underlying Pressure Horizon
}

\author{
Bereslavsky EN* \\ Department of Physics and Mathematics, Russia
}

ISSN: 2637-8035

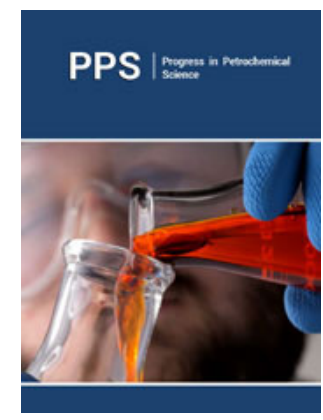

*Corresponding author: Bereslavsky EN, Department of Physics and Mathematics, Russia

Submission: 監 May 25, 2020

Published: 此July 01, 2020

Volume 3 - Issue 3

How to cite this article: Bereslavsky EN. On the Regime of Ground Water during Filtration from Channels in the Soil Layer with the Underlying Pressure Horizon. Progress Petrochem Sci. 3(3). PPS. 000563. 2020.

DOI: $10.31031 /$ PPS.2020.03.000563

Copyright@ Bereslavsky EN, This article is distributed under the terms of the Creative Commons Attribution 4.0 International License, which permits unrestricted use and redistribution provided that the original author and source are credited.

\section{Opinion}

\section{Summary}

In the hydrodynamic formulation, the problem of liquid filtration from a channel filled with water through a soil layer with an underlying pressure horizon of relatively high permeability in the presence of evaporation from the free surface of groundwater is solved. When considering flows from channels, it is usually assumed [1-5]. that filtration occurs only through their bottom, which is usually taken as a horizontal segment. Taking into account the influence of the depth of water in the channels, that is, the study of movement not only through the bottom, but also through the slopes of the channels makes an additional angular special point in the physical area, which significantly complicates the solution of the problem. In this paper, the method developed earlier [6] is used to study the regime of ground water when filtering from such channels filled with water, in the presence of evaporation from a free surface.

In the framework of the theory of plane steady filtration of an incompressible fluid according to Darcy's law deals with the flow of rectangular channel of width 21 with water depth $\mathrm{H}$ in the soil capacity $\mathrm{T}$, underlain by the well permeable pressurized horizon is relatively high permeability, the pressure of which is equal to $\mathrm{HO}(0<\mathrm{HO}<\mathrm{T})$, in the presence of a uniform intensity of evaporation $\varepsilon(0<\varepsilon<1)$ with a free surface. To study the flow formulates a combined multi-parameter boundary problem of the theory of analytic functions which is solved using the method of Polubarinova Kochina PY [1-5], based on the application of the analytic theory of linear differential equations of Fuchs class [7] and the method of integrating such equations with four regular singularities [8-10], which is characteristic for problems of underground hydromechanics [11-15]. It should be noted that taking into account the specific features of the movements under consideration allows you to get solutions to problems in a closed form through elementary functions, which makes its use the most simple, convenient and effective.

On the basis of the model the algorithm of calculation of the size of the zone of saturation and the desired filtration flow rate in the case where filtering of the channels have to evaluate the joint impact on the pattern of such important factors as the filtration capacity of the reservoir and backwater from the deep artesian horizon of relatively high permeability, channel width and depth of the water in the evaporation from the free surface of groundwater. On the basis of the obtained exact analytical dependences and by means of numerical calculations, the hydrodynamic analysis of the influence of all the specified physical parameters of the studied model is carried out. We compare the results of mathematical modeling with the same 
filtration characteristics for the flow pattern in the case when $\mathrm{H}=0$, that is, when filtering only through the bottom of the channels.

\section{References}

1. Polubarinova Kochina PY (1977) Theory of ground water movement. In: Gostekhizdat, Nauka (Eds)., $2^{\text {nd }}$ edn., Moscow, pp. 664-676.

2. Aravin VI, Numerov SN (1953) Theory of motion of liquids and gases in a non-deformable porous medium. Gostekhizdat, Moscow, pp. 616.

3. Development of research on filtration theory in the USSR (1917-1967). Nauka, Moscow, pp. 545.

4. Mikhailov GK, Nikolaevsky VN (1970) Movement of liquids and gases in porous media. Mechanics in the USSR for 50 years. Nauka, Moscow 2: $585-648$.

5. Kochina PY (1991) Hydrodynamics and filtration theory. Selected works. Nauka, Moscow, pp. 351.

6. Bereslavsky EN (2013) On the application of the equations of the fuchs class to calculate seepage from channels and sprinklers. Journal of Applied mathematics and mechanics 77(5): 711-724.

7. Golubev VV (1950) Lectures on the analytical theory of linear differential equations. Leningrad, Gostekhizdat, Moscow, p. 436.

8. Bereslavsky EN (1989) On integration in a closed form of a class of fuchsian equations and its application. Differential equations 25(6): 1048-1050.
9. Bereslavsky EN (1997) On differential equations of the Fuchs class related to the conformal mapping of circular polygons in polar grids. Differential equations 33(3): 292-297.

10. Bereslavsky EN (2010) On accounting for infiltration or evaporation from a free surface by the method of circular polygons. Applied mathematics and mechanics 74(2): 239-251.

11. Bereslavsky EN, Kochina PY (1992) On some equations of the Fuchs class in hydro-aerodynamics. Proceedings of the Russian Academy of Sciences. Fluid and gas mechanics 5: 3-7.

12. Bereslavsky EN, Kochina PY (1997) On differential equations of the Fuchs class occurring in some problems of fluid mechanics proceedings of the Russian Academy of Sciences. Fluid and gas mechanics 5: 9-17.

13. Bereslavsky EN, Kochina PY, Kochina NN (1996) Analytical theory of linear differential equations of the Fuchs class and some problems of underground hydromechanics. $1^{\text {st }}$ edn., Preprint No 567. Institute of problems of mechanics of the Russian Academy of Sciences, Moscow, pp. 122 .

14. Bereslavsky EN (2010) Modeling of filtration flows from channels Reports of the Russian Academy of Sciences 34(4): 472-475.

15. Bereslavsky EN (2011) Modeling of filtration flows from channels. Applied mathematics and mechanics 75(4): 563-571. 